\title{
Magnetic properties engineering of nanopatterned cobalt antidot arrays
}

February 17, 2016

\author{
Andreas Kaidatzis ${ }^{1}$, Rafael P. del Real ${ }^{2}$, Raquel Alvaro ${ }^{3}$, Juan Luis Palma ${ }^{4}$, \\ José Anguita ${ }^{3}$, Dimitrios Niarchos ${ }^{1}$, Manuel Vázquez ${ }^{2}$, Juan Escrig ${ }^{4}$, and \\ José Miguel García-Martín ${ }^{3}$ \\ ${ }^{1}$ Institute of Nanoscience and Nanotechnology, NCSR Demorkitos, 15310 \\ Aghia Paraskevi, Athens, Greece \\ ${ }^{2}$ ICMM-Instituto de Ciencia de Materiales de Madrid (ICMM-CSIC), Sor \\ Juana Inés de la Cruz 3, E-28049 Cantoblanco, Madrid, Spain \\ ${ }^{3}$ IMM-Instituto de Microelectrónica de Madrid (CNM-CSIC), Isaac Newton \\ 8, PTM, E-28760 Tres Cantos, Madrid, Spain \\ ${ }^{4}$ Departamento de Física, Universidad de Santiago de Chile (USACH) and \\ Center for the Development of Nanoscience and Nanotechnology (CEDENNA), \\ Avda. Ecuador 3493, 917-0124, Santiago, Chile \\ Corresponding author: a.kaidatzis@inn.demokritos.gr
}

\begin{abstract}
We report on the study of arrays of $60 \mathrm{~nm}$ wide cobalt antidots, nanopatterned using focused ion beam milling. Square and hexago-
\end{abstract}


nal symmetry arrays have been studied, with varying antidot densities and lattice constant from 150 up to $300 \mathrm{~nm}$. We find a strong increase of the arrays' magnetic coercivity with respect to the unpatterned film, which is monotonic as the antidot density increases. Additionally, there is a strong influence of the array symmetry to the in-plane magnetic anisotropy: square arrays exhibit fourfold symmetry and hexagonal arrays exhibit sixfold symmetry. The above findings are corroborated by magnetic imaging and micromagnetic modeling, which show the magnetic structure of the arrays to depend strongly on the array morphology.

Keywords: Co antidot arrays, focused ion beam, MOKE magnetometry, AFM, MFM, micromagnetic modeling

\section{Introduction}

Magnetic antidot arrays are groups of ordered holes created on a continuous magnetic film. They are being intensively investigated as candidates for highdensity storage media $[1,2]$, magnonic crystals with potential application in microwave devices [3, 4], magnetically-active plasmonics [5], and lately for magnetic biosensing applications [6].

The main non-material-dependent parameters that influence the array magnetic properties are its symmetry and lattice constant and the antidot shape and size. Antidot arrays studies have been mainly focused on square or hexagonal symmetry arrays of circular antidots [7-9], although there are as well studies of other symmetries, like e.g. tetragonal [10] or honeycomb [7] and of square-shaped antidots [11].

Most of the antidot array studies concern $\mu \mathrm{m}$ - and sub- $\mu \mathrm{m}$-scale antidots, fabricated by patterning methods like e-beam lithography $[2,11,12]$ and UV lithography [13]. On the other hand, the principal means for attaining nm-scale antidot arrays are various self-assembly techniques employing porous anodic alumina [8,14], colloidal lithography [15], block copolymer templates [16], or nanochannel glass [17]. However, there are significant inherent drawbacks in all of the self-assembly fabrication methods, mainly regarding the limitations of the array symmetry and/or size and the extent of 
the symmetric domains, which is on the order of some $\mu \mathrm{m}$. Only recently advanced fabrication techniques have been introduced for the study of nm-scale antidot arrays, as for example the use of focused ion beam (FIB) patterning [18], which allow for obtaining antidot arrays of any symmetry extending over areas of several tens of $\mu \mathrm{m}$ or even $\mathrm{mm}$.

In this article, we present our work on nm-scale antidot arrays fabricated using FIB nanopatterning. Magneto-optical Kerr effect (MOKE) magnetometry has been used to characterize the magnetic properties of the arrays. We find an intense increase of the magnetic coercivity $\left(H_{C}\right)$ of the film after patterning, while there is also a monotonic increase of $\mathrm{Hc}$ as the density of nanoholes increases. Additionally, the in-plane anisotropy axes of the patterned film depend strongly on the array symmetry, with alternating hard and easy axes following the fourfold symmetry of the square arrays, or the sixfold symmetry of the hexagonal arrays. Finally, high-resolution magnetic imaging and micromagnetic modeling have been employed and we find the arrays magnetic structure to be commensurate to the array morphology.

\section{Methods}

For sample fabrication, first a continuous $\operatorname{Ti}(2 \mathrm{~nm}) / \mathrm{Co}(10 \mathrm{~nm}) / \mathrm{Au}(10 \mathrm{~nm})$ stack was sputter-deposited in an ultra-high vacuum chamber (base pressure $1 \cdot 10^{-10}$ Torr) on a monocrystalline (0001) sapphire substrate from MaTecK $\mathrm{GmbH}$. The substrate was rotated around its normal axis during the deposition in order to avoid the formation of a strong magnetic anisotropy during the growth of the film. The Ti underlayer promotes adhesion of the stack, while the Au capping layer protects Co from oxidation.

Antidot arrays were directly etched on the continuous stack using an IonLine FIB machine, with $30 \mathrm{keV}$ Ga ions and $6.9 \mathrm{pA}$ ion current. For each lattice position of the array, the stack was exposed to the focused beam for 0.17 seconds, thus etching each one of the antidots. The exposure time corresponds to a dose of $1.2 \mathrm{pC}$, that was enough to etch the sample at a depth of $42 \mathrm{~nm}, 20 \mathrm{~nm}$ more than the whole metallic stack thickness.

Atomic/Magnetic Force Microscopy (AFM/MFM) measurements have been performed using a Bruker Dimension Icon microscope and commer- 
cial AFM/MFM probes (Bruker MESP). MFM images were obtained using the phase imaging double-pass tapping-mode: surface topography is recorded during the first pass and then the tip is lifted at a certain height and the magnetic contrast is recorded. As the MFM tip is magnetized along the axis vertical to the sample, the observed contrast originates from magnetic charges on the sample surface. All microscopy images were processed using the WSxM software [19].

Longitudinal MOKE hysteresis loops of the arrays were obtained using a Nanomoke 2.0 (Durham Magnetooptics Ltd.) setup, using $635 \mathrm{~nm}$ laser wavelength. The laser spot was focused to a $3 \mu \mathrm{m}$ diameter. The spot was placed into each antidot array using the robotic motion stage which allows the movement of the sample with a precision of $1 \mu \mathrm{m}$. In order to check that the laser spot is placed in the right position, the longitudinal reflectivity was measured. Besides, the focused laser spot and the sample surface can be viewed together. The sample was rotated using a rotation stage and the laser spot was reallocated using the same procedure above mentioned. Magnetic field was applied along the $0^{\circ}, 45^{\circ}$, and $90^{\circ}$ direction when measuring the square arrays and along $0^{\circ}, 30^{\circ}, 60^{\circ}$ and $90^{\circ}$ direction when measuring the hexagonal arrays (see figure 1 ).

Micromagnetic modeling has been performed using the GP Magnet program $^{1}$ software that allows us to simulate three-dimensional systems of large volume using graphic processing units. Under this frame, the ferromagnetic system is divided into cubic cells with a uniform magnetization inside each cell. For the calculations we have considered a circular sample (diameter $8 \mu \mathrm{m})$ of Co antidots defined by a thickness of $8 \mathrm{~nm}$ and a pore diameter of $55 \mathrm{~nm}$, which are arranged in a square array with a lattice constant of $300 \mathrm{~nm}$. In order to obtain an adequate description for the Co sample, the size of the cubic cell has to be smaller than the exchange length of the material, defined by $[20] \Delta_{d}=\sqrt{2 A / \mu_{0} M_{S}^{2}}=4.7 \mathrm{~nm}$, where $\mathrm{A}=30 \times 10^{-12} \mathrm{~J} / \mathrm{m}$ is the exchange stiffness constant and $M_{S}=1400 \times 10^{3} \mathrm{~A} / \mathrm{m}$ is the saturation magnetization. Thus, we have chosen a cell size of $4 \times 4 \times 4 \mathrm{~nm}^{3}$, which implies that the micromagnetic simulations involve 8 million cells and 1108 antidot elements. Additionally, we included a small uniaxial anisotropy of

\footnotetext{
${ }^{1}$ GoParallel S.L., http://www.goparallel.net/index.php/gp
} 
$2400 \mathrm{~J} / \mathrm{m}^{3}$ in $90^{\circ}$ direction, which was obtained from the experimental measurements (see discussion related to figure 6 (a)). In all cases the damping constant was $\alpha=0.5$.

\section{Results and discussion}

\subsection{Sample morphology}

AFM imaging (see figure 1) reveals that the surface morphology of the initial film has very low roughness (root mean square roughness is $0.1 \mathrm{~nm}$ ), which is desirable for the ulterior FIB nanopatterning.

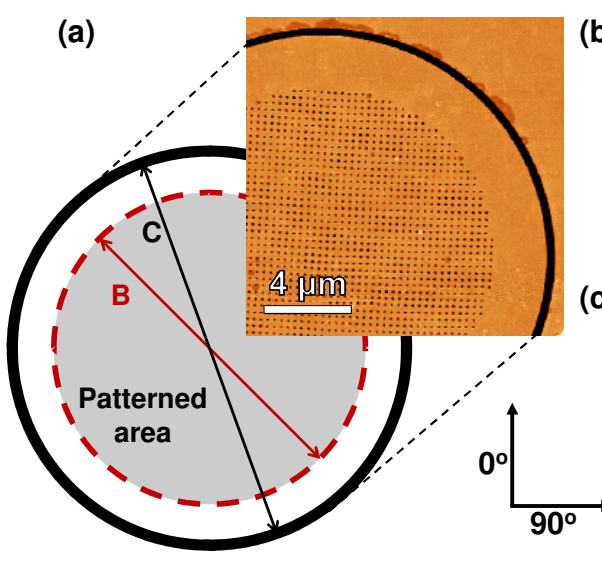

(b)
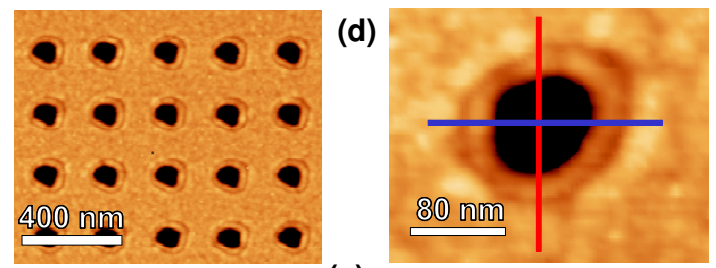

(c)
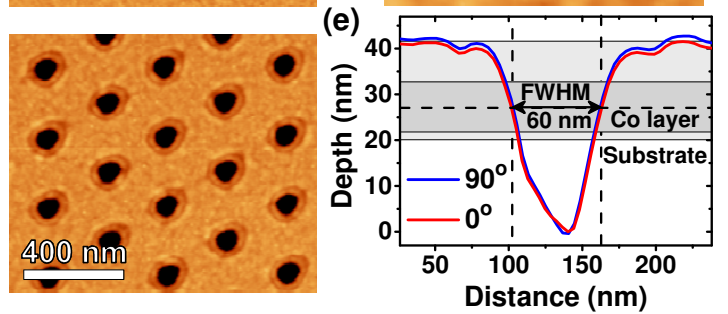

Figure 1: Typical morphology of the arrays obtained by AFM. (a) Design and global view of the arrays: $B$ is $15 \mu \mathrm{m}$ and $C$ is $20 \mu \mathrm{m}$. The color scale (from dark to bright) is from 0 to $45 \mathrm{~nm}$. Typical images of square (b) and hexagonal (c) arrays. The color scale is from 0 to $15 \mathrm{~nm}$. (d) Typical antidot morphology obtained at image (c). The color scale is from 0 to $12 \mathrm{~nm}$. Straight lines indicate directions where line profiles are obtained and shown in (e). All the samples share the same coordinates, as-defined in this figure.

After patterning each antidot array has the shape of a disk (diameter $15 \mu \mathrm{m}$ ), for avoiding any shape anisotropy, surrounded by a circular trench that magnetically decouples the array from the rest of the layer (see figure 1 
(a)). The trench was etched using a $21 \mathrm{mC} / \mathrm{cm}^{2}$ ion beam dose for obtaining a depth equal to the one of the antidots. Moreover, for the sake of comparison, regions confined by a trench but without any patterning were also prepared, i.e. defining Co disks with $20 \mu \mathrm{m}$ diameter.

The studied symmetries are square and hexagonal (see figures 1 (b) and 1 (c)). From a magnetic viewpoint each nanohole may be considered as a defect, since they act as pinning centers for the domain-wall (DW) motion during magnetization reversal [21]. If $a$ is the lattice constant (nearest neighbor centre-to-centre distance) and $d$ the hole diameter, the defect volume density (DVD), which is the ratio of the surface covered by holes to the total surface, is given by $\frac{\pi}{4}\left(\frac{d}{a}\right)^{2}$ for the square arrays and by $\frac{\pi}{2 \sqrt{3}}\left(\frac{d}{a}\right)^{2}$ for the hexagonal ones.

The diameter of the antidots is determined at figure 1 (e) as the full-width at half maximum of the hole, at the level of the Co layer, and it is measured to be $60 \mathrm{~nm}$. The antidot appears to have a "tapered" geometry, however, this is due to the convolution with the AFM tip, which has a pyramid geometry with $25^{\circ}$ slope. Four different arrays were etched for each symmetry, having lattice constant equal to $150,200,250$, and $300 \mathrm{~nm}$. The DVD is $0.126,0.071$, 0.045 and 0.031 for the square arrays and $0.145,0.082,0.052$ and 0.036 for the hexagonal ones, respectively.

A striking characteristic of sample morphology is the apparent "rim" around the antidots: this appears as a $2.5 \mathrm{~nm}$ depression when approaching the antidot center, followed by a $1 \mathrm{~nm}$ elevation, while its average width is $18 \mathrm{~nm}$. This pattern is consistently repeated around every antidot studied. It is interesting to note that a similar effect has been previously reported on FIB etched antidots and shown to be a local film alteration due to the tail of the Gaussian-like section of the ion beam [18].

\subsection{Hysteresis curves}

Figure 2 (a) shows MOKE loops obtained at an unpatterned Co disk along the $0^{\circ}$ and $90^{\circ}$ directions. It is clear that the unpatterned film has a small uniaxial in-plane magnetic anisotropy; the easy axis (EA) is along $90^{\circ}$, with $H_{C}=21$ Oe and an almost square loop: the remanence magnetization to sat- 


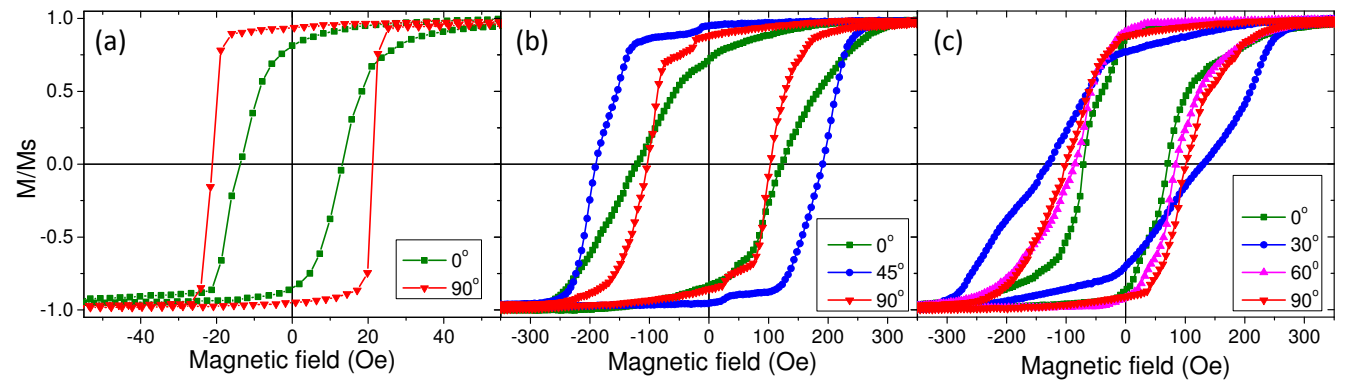

Figure 2: Representative MOKE loops obtained along various magnetic field directions. (a) Unpatterned area confined by a circular trench. (b) Square array. (c) Hexagonal array. In both cases the lattice constant is $250 \mathrm{~nm}$, resulting in respective defect volume density values of 0.071 and 0.082 . Please note the smaller magnetic field axis scale used in (a).

uration magnetization $\left(M_{R} / M_{S}\right)$ value is 0.95 . The $0^{\circ}$ direction is the hard axis (HA), as $H_{C}$ is 14 Oe and $M_{R} / M_{S}$ is 0.82 . However, significant hysteresis is observed, in conjunction to the non-epitaxial polycrystalline structure of the films; in the past it has been shown that vacuum deposited polycrystalline Co films have hcp crystallites, not completely randomly oriented, that induce an in-plane uniaxial anisotropy [22]. In our case, the mono-crystalline sapphire substrate should be additionally taken into account, as it probably induces a preferred crystallites texture, in spite of the fact that it was rotating during deposition.

Figure 2 (b) shows representative square array MOKE loops (lattice constant is $250 \mathrm{~nm}$ and DVD is 0.071 ). The increase of $H_{C}$ with respect to the unpatterned film is pronounced. Small steps appear at low field, i.e. of the same magnitude as the $H_{C}$ values of the unpatterned film. This suggests a facile DW motion between antidots (at the unpatterned regions of the array) and strong DW pinning at antidots, indicating a combined effect of the uniaxial magnetocrystalline anisotropy of the unpatterned film and antidot array symmetry.

The $45^{\circ}$ direction (second-nearest-neighbor) is the magnetic anisotropy EA ( $H_{C}$ is 186 Oe and $M_{R} / M_{S}$ is 0.95$)$. The $0^{\circ}$ direction (nearest-neighbor) is a HA $\left(H_{C}\right.$ is 120 Oe and $M_{R} / M_{S}$ is 0.78$)$ and it coincides with the unpat- 
terned film HA. However, although the $90^{\circ}$ direction is also along nearestneighbors, $H_{C}$ is smaller (100 Oe) but $M_{R} / M_{S}$ is higher (0.88). This could be attributed to a convolution effect with the unpatterned film EA, which lies along the $90^{\circ}$ direction; in the past it has been shown that a small inplane uniaxial anisotropy may greatly influence magnetization reversal [23]. It should be noted that similar results are obtained for the rest of the square symmetry arrays. Finally, a similar behavior has been also reported in the past for square symmetry Co antidot arrays [9], although it concerned $\mu \mathrm{m}-$ scale antidots and only one antidot density was studied.

Figure 2 (c) shows representative hexagonal array MOKE loops (lattice constant is $250 \mathrm{~nm}$ and DVD is 0.082 ). There is also a multi-fold increase of $H_{C}$ with respect to the unpatterned film. The lowest $H_{C}$ is obtained at $0^{\circ}$ (66 Oe), a nearest-neighbor direction, whereas the highest at $30^{\circ}$ (129 Oe), a second-nearest neighbor direction. Intermediate $H_{C}$ values are obtained at $60^{\circ}$ (85 Oe) and at $90^{\circ}$ (98 Oe).

From MOKE loops, the $H_{C}$ and $M_{R} / M_{S}$ values are determined for all samples and shown in figure 3 as a function of the applied magnetic field direction. In most cases there is a clear dependence of $H_{C}$ and $M_{R} / M_{S}$ on direction and the same trend is usually maintained for a given array symmetry. For square arrays, the maximum $H_{C}$ and $M_{R} / M_{S}$ values are obtained along the $45^{\circ}$ direction (second-nearest-neighbors), indicating that this is a magnetic anisotropy EA. The only exception is the array with the lowest DVD (0.031), where a monotonic decrease of $H_{C}$ and increase of $M_{R} / M_{S}$ is observed, suggesting that for the lowest DVD the array magnetic anisotropy is dominated by the unpatterned film uniaxial anisotropy.

For hexagonal arrays, it is generally observed that $H_{C}$ and $M_{R} / M_{S}$ have an opposite variation trend making the interpretation of data challenging. The maximum $H_{C}$ values are along $30^{\circ}$ and $90^{\circ}$ directions (second-nearestneighbors), except for the array with maximum DVD (0.145) where the inverse trend is observed. On the contrary, $M_{R} / M_{S}$ values have local maxima along $0^{\circ}$ and $60^{\circ}$ directions (nearest-neighbors). The only exception is the array with minimum DVD (0.036) where a monotonic increase is observed, again indicating that the array magnetic anisotropy is dominated by the unpatterned film uniaxial anisotropy. 


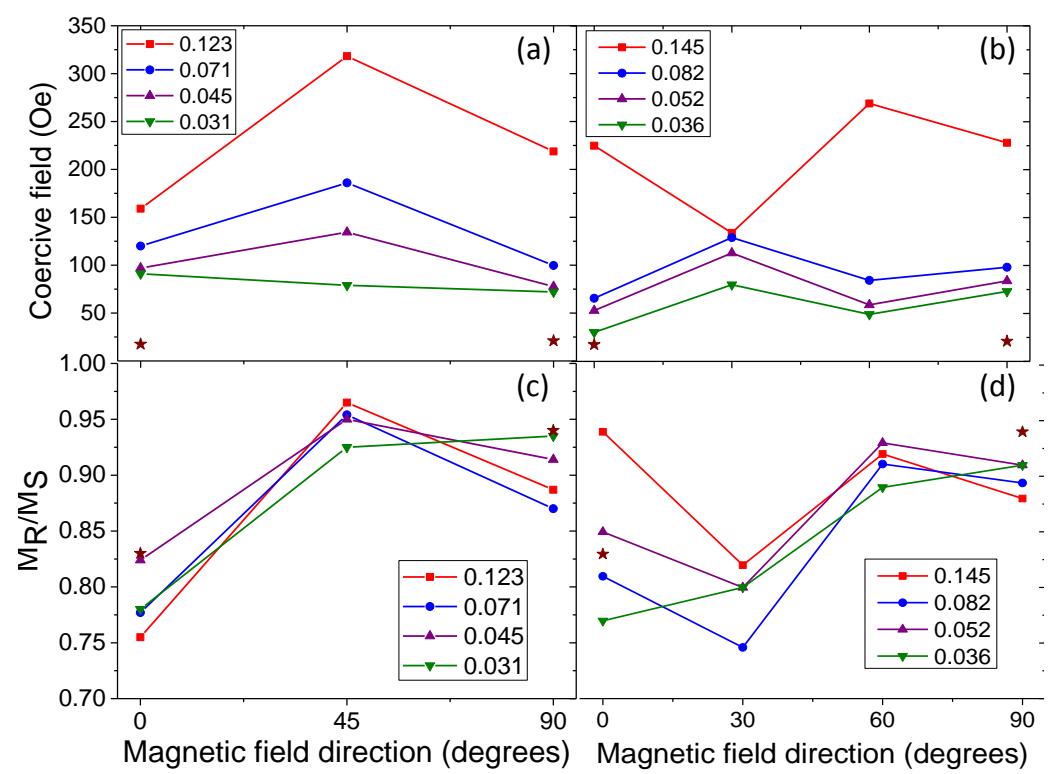

Figure 3: $H_{C}$ values as a function of magnetic field direction for (a) square and (b) hexagonal symmetry arrays. $M_{R} / M_{S}$ values for (a) square and (b) hexagonal symmetry arrays. Data for various defect volume density (DVD) values are shown. Brown stars indicate the values obtained at an unpatterned area confined by a circular trench.

It is worth pinpointing that the remanent magnetization barely depends on DVD, whereas the coercivity strongly depends on it. This behavior indicates that the separation between holes does not affect the type of domain walls that are formed, but it strongly alters their pinning during reversal. In fact, the MFM experiments that will be discussed later showed the same type of domain walls for all the arrays with the same symmetry.

In order to highlight the importance of DVD on the strength of the pinning, figure 4 shows all the $H_{C}$ data obtained as a function of DVD. For both array symmetries, there is a monotonic increase of $H_{C}$ as DVD increases. The above are in accordance with previous studies which predict an increase of $H_{C}$ as the density of domain-wall pinning centers (in this case the antidots) increases [21]. The maximum $H_{C}$ value for both symmetries is obtained at the maximum DVD value and it is 318 Oe for the square symmetry arrays 
at $45^{\circ}$ and 268 Oe for the hexagonal symmetry at $60^{\circ}$.

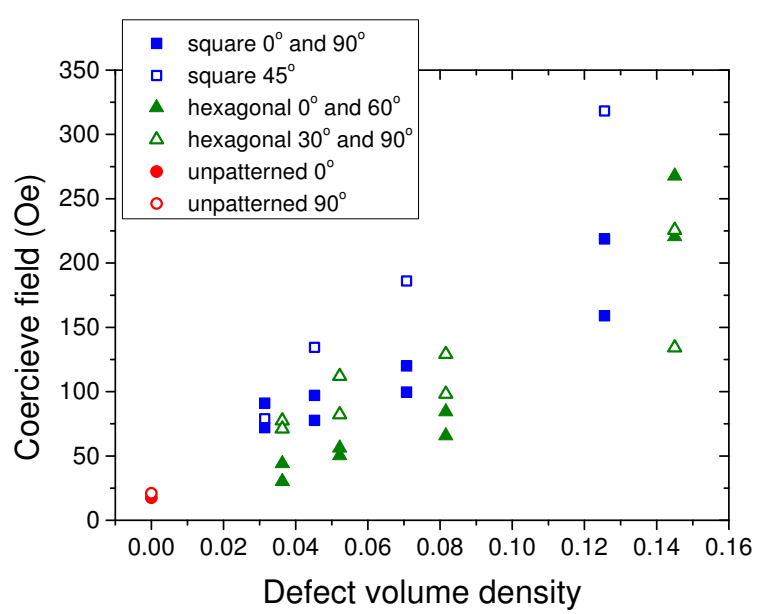

Figure 4: Variation of $H_{C}$ with respect to defect volume density. Square arrays: field direction along first neighbors (filled squares) and second neighbors (open squares). Hexagonal arrays: field direction along first neighbors (filled triangles) and second neighbors (open triangles). Unpatterned area confined by trench: field direction along $0^{\circ}$ (filled circle) and $90^{\circ}$ (open circle).

The arrays with the lowest DVD exhibit small $H_{C}$ dispersion, indicating that at low DVD values antidot density prevails over antidot symmetry in tailoring film magnetic properties. However, as DVD increases, the array symmetry becomes more important: $H_{C}$ values are more dispersed and the maximum value is always at the second-nearest-neighbor direction, for both symmetries.

The only exception to the above-mentioned tendency appears at the hexagonal array with the highest DVD, which exhibits opposite behavior: the $60^{\circ}$ direction has the highest $H_{C}$, while at $0^{\circ} H_{C}$ is almost equal to $90^{\circ}$. This could be attributed to the onset of a "crossover" from antidot (continuous magnetic film with an array of holes) to dot (an array of magnetically isolated nanostructures) magnetic behavior previously reported [18]; it has been shown that for square symmetry array and DVD values higher than 0.130 , despite the fact that the film is still magnetically continuous, emerges an intermediate regime between antidot and dot, due to the close proximity 
of neighboring regions affected by the tail of the Gaussian-like section of the ion beam. It should be noted that although we try to interpret the behavior of hexagonal symmetry arrays based on findings concerning square symmetry arrays, the study presented in reference [18] takes into account only DVD, without considering the effect of symmetry or applied field direction. Thus, it could be assumed that this "crossover" effect is more pronounced along second-nearest-neighbor directions at hexagonal arrays $\left(30^{\circ}\right.$ and $\left.90^{\circ}\right)$, resulting in the observed behavior.

\subsection{Magnetic imaging and micromagnetic modeling}

MFM imaging has been performed for obtaining microscopic insight of the arrays magnetic structure. All images show the magnetic structure of the arrays in remanence state: first a $15 \mathrm{kOe}$ magnetic field is applied along a given direction in order to magnetically saturate the sample and then the field is reduced to zero before imaging.

Figure 5 (a) shows the typical magnetic structure of an unpatterned Co layer confined by circular trench. Magnetic domains have random structure and sizes from several tens to hundreds of $\mathrm{nm}$, much larger than the average grain size, indicating strong magnetic coupling between grains. Arrows indicate abrupt signal changes, which correspond to DW drag effects because of the magnetic stray field of the MFM tip, which is in coherence with the low $H_{C}$ value of the unpatterned film.

Figure 5 (b) shows the typical magnetic structure of a $200 \mathrm{~nm}$ hexagonal array at remanence state, after applying magnetic field along the $30^{\circ}$ direction. It is immediately clear that the magnetic structure is commensurate to the morphology of the array. Dark contrast stripes correspond to super-DWs [12] and they are aligned along the $0^{\circ}$ and $120^{\circ}$ (equivalent to $60^{\circ}$ ) directions. As before, there is significant DW dragging due to the MFM tip stray field, although $H_{C}$ is 113 Oe, much higher than the unpatterned film. It should be noted that similar magnetic structure is observed for the rest of the arrays and applied field directions, suggesting that this is the equilibrium magnetic structure of the hexagonal arrays. This absence of discrepancy between directions is attributed to the non-perfect alignment of 

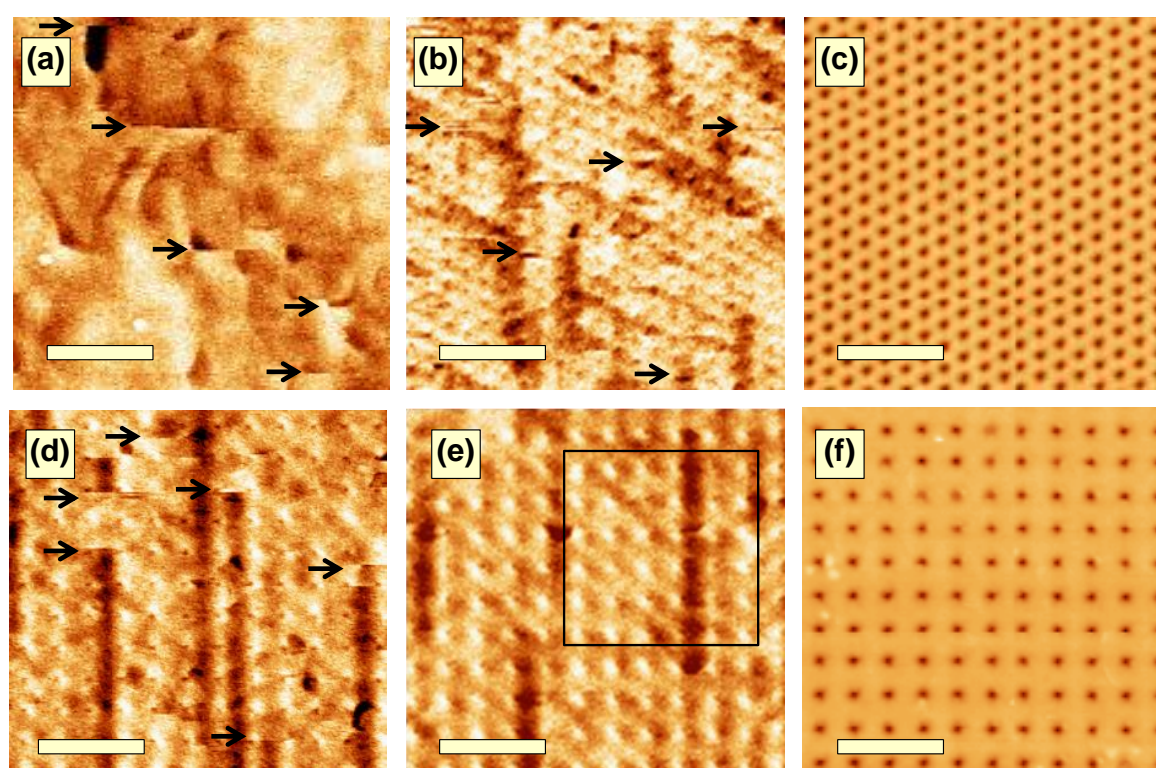

Figure 5: MFM images of the samples' magnetic structure. (a) Unpatterned Co layer confined by circular trench. (b) Hexagonal array (200 $\mathrm{nm}$ period), magnetic field along $30^{\circ}$, and (c) the corresponding AFM image. Square array (300 nm period): (d) magnetic field along 90 and (e) magnetic field along $45^{\circ}$ (the square frame indicates the simulated area shown in figure 6), and (f) the corresponding AFM image. The scale bar is $1 \mu \mathrm{m}$ and all figures have the same area. The color scale at the MFM images is from 0 to $0.9^{\circ}$ and at the AFM images is from 0 to $25 \mathrm{~nm}$. Lift height during MFM imaging was always $40 \mathrm{~nm}$.

the sample during the magnetization process before MFM imaging and the subtle variation between alternating EAs and HAs.

Figure 5 (c) shows the magnetic structure of the $300 \mathrm{~nm}$ square symmetry array, after applying magnetic field along the $90^{\circ}$ direction. In this case only vertical super-DWs are apparent, which are commensurate to the square symmetry of the array. As before, there are several DW-drag events, although $H_{C}$ is 72 Oe. Figure 5 (d) shows the magnetic structure of the $300 \mathrm{~nm}$ square symmetry array, after applying magnetic field along the the $45^{\circ}$ direction. Apparently there is some distinction from the $90^{\circ}$ case: the image has mixed dark and bright contrast aligned along the $45^{\circ}$ directions, with only a few 
vertical super-DWs. The overall contrast is mainly bright, in accordance to the high remanence magnetization obtained from the MOKE loops for magnetic field applied along the $45^{\circ}$ direction. The most striking difference is the total absence of DW-drag: although $H_{C}$ is 79 Oe, only slightly higher than the $90^{\circ}$ direction and much lower than the hexagonal symmetry array imaged in figure 5 (c), the stray field from the MFM tip does not affects the array magnetic structure.

In order to understand more deeply the magnetic structure of the arrays and the formation of super-DWs, micromagnetic simulations were performed [24]. Simulations consider an external magnetic field applied at $45^{\circ}$, from saturation (10 kOe) to -30 Oe, passing through the remnant state (0 Oe).

When a $10 \mathrm{kOe}$ field is applied, all magnetic moments are pointing in the same direction as the external field $\left(45^{\circ}\right)$. Thus, it can be said that the sample is saturated (see figure $6(\mathrm{~b})$ ). Then, when the external magnetic field is set to $\mathrm{O}$ Oe (figure 6 (c)), most of the magnetic moments relax to the direction along the easy magnetization axis $\left(90^{\circ}\right)$ defined by the small magnetic anisotropy measured experimentally. However, those magnetic moments closest to the edge of the sample remain pinned at $45^{\circ}$ because of the edge effect (the edge tries to align magnetic moments in $0^{\circ}$, while the magnetocrystalline anisotropy favors the direction of $90^{\circ}$. Thus, on average, the magnetic moments will remain pinned at $45^{\circ}$. Once a field of -10 Oe is applied at $45^{\circ}$, the magnetic moments closest to the surface remain pinned at $45^{\circ}$ while the inner magnetic moments reverse their magnetization, thereby forming a soft U-shaped magnetization (figure $6(\mathrm{~d})$ ). If the field is further reduced to -20 Oe, there is simply an increase of the angle at which the magnetic moments face each other in the U-shaped magnetization (figure 6 (e)). Finally, at a field of -30 Oe, the magnetic moments generate $180^{\circ}$ superDWs (figure 6 (f)), corresponding to the magnetic contrast observed in the MFM images. Thus, micromagnetic simulations intend to give a qualitative explanation of how patterning a thin film affects the formation of magnetic domains on its surface. From these simulations we can conclude that an antidot pattern created on a thin film induces linear magnetic domains. 

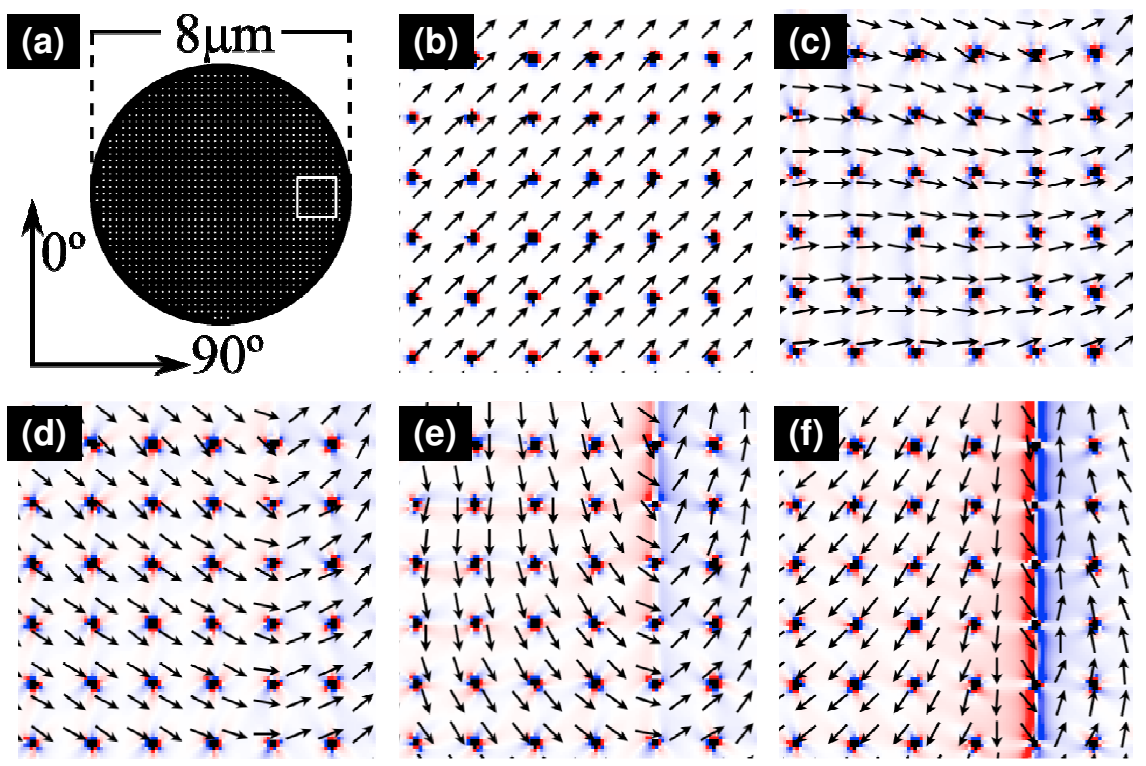

Figure 6: Simulated magnetic structure of the array samples. a) Circular sample of $8 \times 8 \mu \mathrm{m}^{2}$ of Co antidots defined by a thickness of $8 \mathrm{~nm}$ and a pore diameter of $55 \mathrm{~nm}$, which are arranged in a square array with a lattice constant of $300 \mathrm{~nm}$. The axes show the directions according to the experimental results. The inset white square corresponds to the area shown in the following figures. The external magnetic field is applied at $45^{\circ}$ with an intensity of b) $10 \mathrm{kOe}, \mathrm{c}) 0$ Oe, d) -10 Oe, e) -20 Oe and f) -30 Oe, in order to reproduce the formation of super-DWs in the remnant state. The color scale (red-white-blue) corresponds to divergence from -0.02 to 0.02.

\section{Conclusions}

FIB has allowed for fabricating antidot arrays with $60 \mathrm{~nm}$ hole diameter and long-range order, following two symmetries: square and hexagonal. Various antidot-to-antidot distances have been studied for each symmetry. We have shown the important effects of antidot array symmetry and defect volume density on the coercivity and anisotropy of magnetic thin films: coercivity increases with the density of antidots and magnetic anisotropy axes strongly depend on array symmetry. MFM images show the magnetic structure of the arrays to be commensurate to their morphology, while micromagnetic 
simulations indicate the mechanisms of super-DW creation in magnetic antidot arrays. The above findings provide further insight for fine tuning the magnetic properties of thin films employing arrays of antidots.

\section{Acknowledgments}

A.K. received funding from the EC through a FP7 individual Marie Curie fellowship (Grant No. 272470). We also acknowledge funding from the EC through a FP7-ICT project (Grant No. 318144), from CSIC (ref. iLINK0783), from Comunidad de Madrid (ref: Nanofrontmag), from DICYT, from Fondequip (ref. EQM140092), from Fondecyt (refs. 3130393 and 1150952), and from the Basal Project (ref. FB0807).

\section{References}

[1] R. P. Cowburn, A. O. Adeyeye, and J. A. C. Bland. Appl. Phys. Lett., 70:2309, 1997.

[2] L. J. Heyderman, F. Nolting, D. Backes, S. Czekaj, L. Lopez-Diaz, M. Kläui, U. Rüdiger, C. A. F. Vaz, J. A. C. Bland, R. J. Matelon, U. G. Volkmann, and P. Fischer. Phys. Rev. B, 73:214429, 2006.

[3] V V Kruglyak, S O Demokritov, and D Grundler. J. Phys. D: Appl. Phys., 43:264001, 2010.

[4] A. Barman. J. Phys. D: Appl. Phys., 43:195002, 2010.

[5] J.B. González-Díaz, J.M. García-Martín, A. García-Martín, D. Navas, A. Asenjo, M. Vázquez, M. Hernández-Vélez, and G. Armelles. Appl. Phys. Lett., 94:263101, 2009.

[6] P. J. Metaxas, M. Sushruth, R. A. Begley, J. Ding, R. C. Woodward, I. S. Maksymov, M. Albert, W. Wang, H. Fangohr, A. O. Adeyeye, and M. Kostylev. Appl. Phys. Lett., 106:232406, 2015.

[7] C.C. Wang, A.O. Adeyeye, and N. Singh. Nanotechnology, 17:1629, 2006. 
[8] D. Navas, M. Hernández-Vélez, M. Vázquez, W. Lee, and K. Nielsch. Appl. Phys. Lett., 90:192501, 2007.

[9] N. G. Deshpande, M. S. Seo, X. R. Jin, S. J. Lee, Y. P. Lee, J. Y. Rhee, and K. W. Kim. Appl. Phys. Lett., 96:122503, 2010.

[10] P. Vavassori, G. Gubbiotti, G. Zangari, C. T. Yu, H. Yin, H. Jiang, and G. J. Mankey. J. Appl. Phys., 91:7992, 2002.

[11] D. H. Y. Tse, S. J. Steinmuller, T. Trypiniotis, D. Anderson, G. A. C. Jones, J. A. C. Bland, and C. H. W. Barnes. Phys. Rev. B, 79:054426, 2009 .

[12] X. K. Hu, S. Sievers, A. Müller, V. Janke, and H. W. Schumacher. Phys. Rev. B, 84:024404, 2011.

[13] C. Yu, M. J. Pechan, and G. J. Mankey. Appl. Phys Lett., 83:3948, 2003.

[14] C. T. Sousa, D. C. Leitao, M. P. Proenca, J. Ventura, A. M. Pereira, and J. P. Araujo. Appl. Phys. Rev., 1:031102, 2014.

[15] M. E. Kiziroglou, X. Li, D. C. Gonzalez, C. H. de Groot, A. A. Zhukov, P. A. J. de Groot, and P. N. Bartlett. J. Appl. Phys., 100:113720, 2006.

[16] K. Liu, S. M. Baker, M. Tuominen, T. P. Russell, and I. K. Schuller. Phys. Rev. B, 63:060403, 2001.

[17] R. Cheng, B. L. Justus, A. Rosenberg, D. N. McIlroy, Z. Holman, D. Zhang, and Y. Kranov. J. Appl. Phys., 108:086110, 2010.

[18] C. Castán-Guerrero, J. Herrero-Albillos, J. Bartolomé, F. Bartolomé, L. A. Rodríguez, C. Magén, F. Kronast, P. Gawronski, O. ChubykaloFesenko, K. J. Merazzo, P. Vavassori, P. Strichovanec, J. Sesé, and L. M. García. Phys. Rev. B, 89:144405, 2014.

[19] I. Horcas, R. Fernández, J. M. Gómez-Rodríguez, J. Colchero, J. GómezHerrero, and A. M. Baro. Rev. Sci. Instrum., 78:013705, 2007.

[20] A. Hubert and R. Schäfer. Magnetic domains. Springer, Berlin, 1998. 
[21] H.R. Hilzinger and H. Kronmuller. J. Magn. Magn. Mat., 2:11, 1976.

[22] M. J. Baird, J. A. C. Bland, E. Gu, A. J. R. Ives, F. O. Schumann, and H. P. Hughes. J. Appl. Phys., 74:5658, 1993.

[23] R. P. Cowburn, S. J. Gray, J. Ferré, J. A. C. Bland, and J. Miltat. J. Appl. Phys., 78:7210, 1995.

[24] J.L. Palma, C. Gallardo, L. Spinu, J.M. Vargas, L.S. Dorneles, J.C. Denardin, and J. Escrig. J. Magn. Magn. Mat., 344:8, 2011. 